\title{
The Role of Canaloplasty in the Surgical Treatment of Open-Angle Glaucoma
}

\section{Paolo Brusini*, Claudia Tosoni and Marco Zeppieri}

Department of Ophthalmology, Azienda Ospedaliero-Universitaria "Santa Maria della Misericordia”, Udine, Italy

\begin{abstract}
Canaloplasty is a relatively new surgical option in the treatment of open-angle glaucoma. This non-perforating technique uses a microcatheter connected to a flickering red light laser source, which is inserted within Schlemm's canal. A 10-0 prolene suture is tied to the distal tip of the microcatheter, which is gently guided out of the canal in the opposite direction and then left tensioned while a small amount of viscoelastic agent is injected in Schlemm's canal. The aim of this surgery is to facilitate aqueous outflow through natural pathways, with less risks of post-operative complications.
\end{abstract}

Keywords: Canaloplasty; Non-perforating surgical procedures; Chronic open-angle glaucoma; Schlemm's canal

Canaloplasty is a new surgical technique used to treat various types of open angle glaucoma, which is similar to Stegmann's viscocanalostomy. It is a non-perforating bleb-less technique, which involves positioning a 10-0 prolene suture that is left tensioned within Schlemm's canal that is dilated with the use of viscoelastic. This surgery aims at facilitating aqueous outflow through natural pathways (collector channels and aqueous veins) [1-9].

In brief, surgery involves first forming a fornix-based conjunctival flap, followed by a superficial scleral flap, similar to deep sclerectomy, which is dissected about $1.5 \mathrm{~mm}$ forward. A deep scleral flap is then sculpted, followed by the opening and deroofing of Schlemm's canal. This surgical step is performed after paracentesis to avoid IntraOperatory Intraocular Pressure (IOP) spikes, thus reducing the risk of perforation of the trabeculo-descemet membrane. After deep scleral flap removal, the two ostia of the canal are dilated using high molecular weight hyaluronic acid (Healon GV). A 200 micron microcatheter (iTrack by iScience Interventional, Menlo Park, CA, USA) connected to a laser flickering red light source is used to facilitate the identification of the distal tip through the sclera (Figure 1) while it is inserted and pushed though the entire $360^{\circ}$ of Schlemm's canal until it comes out of the other end of the of the canal opening. Once the microcatheter exits the ostia, a single or double 10-0 prolene suture is tied to the distal tip and then withdrawn and pulled back in the opposite direction through the canal while a small amount of viscoelastic agent is delivered in at

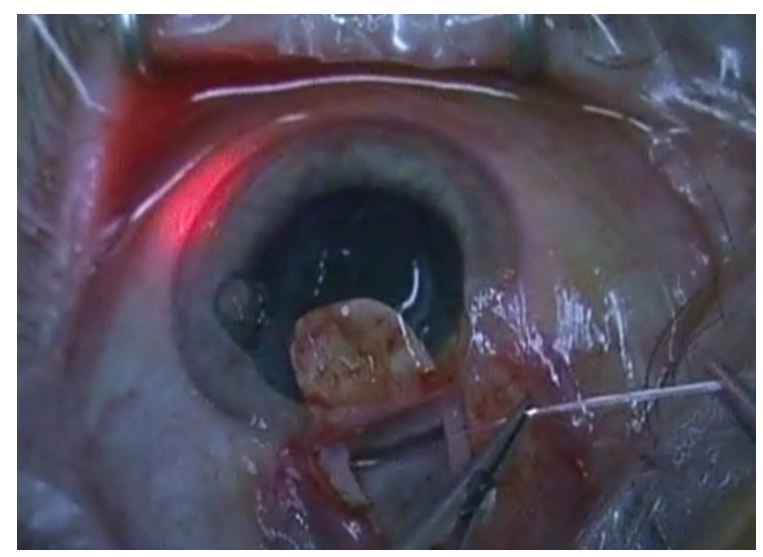

Figure 1: Insertion of the microcatheter within Schlemm's canal during canaloplasty. It is connected to a laser flickering red light source and is used to facilitate the identification of the distal tip while it is inserted and pushed though the entire $360^{\circ}$ of the canal until it comes out of the other end. every two or three clock hours. The suture is then knotted under tension in order to inwardly distend the trabecular meshwork. The superficial scleral flap is then tightly sutured to ensure a watertight closure, thus preventing any bleb formation. The conjunctival flap is then sutured with 10-0 vicryl sutures.

IOP tends to be in the mid teens after surgery, often without any medical therapy. Canaloplasty is an interesting surgical alternative mostly because, unlike traditional trabeculectomy, a filtering bleb is not needed to enhance aqueous outflow. Postoperative results tend to be quite good; after just a few weeks the eye shows little signs of surgery, inflammation or discomfort. Gonioscopy can be used to show that even after several years, the prolene suture remains intact within the canal.

Canaloplasty can be a viable alternative in patients with early to moderate glaucoma, which does not need a low target IOP. The procedure may prove to be ineffective in a small percent of patients, which may be due to a non-reversible collapse of collector channels or other outflow pathways that cannot be enlarged due to anatomical factors.

Canaloplasty is indicated for the following types of patients: 1) primary open-angle glaucoma; 2) pseudoexfoliation glaucoma; and 3) pigmentary glaucoma. Canaloplasty can also be considered in patients with failed trabeculectomy or previous filtrating surgeries, as long as Schlemm's canal has been left undamaged and intact [10].

Canaloplasty should not be considered in: 1) angle-closure glaucoma; 2) narrow-angle glaucoma (unless previous laser or surgical iridectomy); 3) neovascular glaucoma; 4) post-traumatic glaucoma; 5) eyes with damage to Schlemm's canal due to previous ocular surgery or extensive laser trabeculoplasty with peripheral anterior synechiae; 6) ocular hypertension due to increased episcleral venous pressure; and, 7) other forms of secondary glaucomas.

*Corresponding author: Paolo Brusini, Department of Ophthalmology, Azienda Ospedaliero-Universitaria "Santa Maria della Misericordia", p.le S. Maria della Misericordia 15, 33100 Udine, Italy, Tel: ++432-552747; Fax ++432-552741; E-mail: brusini@libero.it

Received December 12, 2013; Accepted January 28, 2014; Published February 05, 2014

Citation: Brusini P, Tosoni C, Zeppieri M (2014) The Role of Canaloplasty in the Surgical Treatment of Open-Angle Glaucoma. Surgery Curr Res 4: 169. doi:10.4172/2161-1076.1000169

Copyright: ( 2014 Brusini $P$, et al. This is an open-access article distributed under the terms of the Creative Commons Attribution License, which permits unrestricted use, distribution, and reproduction in any medium, provided the original author and source are credited. 
Canaloplasty offers several important advantages when compared to traditional trabeculectomy [11,12], which include: 1) no subconjunctival bleb formation; 2) no need for antimetabolites; 3 ) better and faster post-operative healing; 4) simplified and less frequent post-operative follow-ups; 5) limited post-operative complications that are seldom sight threatening; and, 6) postoperative results and IOP tend to be stable over time.

There are some disadvantages to canaloplasty, which include: 1) long and difficult learning curve; 2) necessity of specifically designed (and expensive) instruments; 3 ) average post-operative IOP levels tend not to be very low; and, 4) impossibility to cannulate Schlemm's canal in about $10 \%$ of eyes. Canaloplasty can easily be converted in these cases into either a deep sclerectomy or a viscocanalostomy.

Although canaloplasty is a demanding and rather difficult technique, this surgical procedure tends to provide promising surgical outcomes in patients with various types of open-angle glaucoma. Further multicenter prospective studies that involve a larger group of different types of glaucoma patients randomly selected to undergo canaloplasty versus other traditional glaucoma surgeries are needed to confirm the advantages, efficiency in IOP lowering over time, and limiting complications of canaloplasty. Moreover, the correct indications for canaloplasty need to be further assessed in prospective studies.

\section{References}

1. Lewis RA, von Wolff K, Tetz M, Korber N, Kearney JR, et al. (2007) Canaloplasty: circumferential viscodilation and tensioning of Schlemm's canal using a flexible microcatheter for the treatment of open-angle glaucoma in adults: interim clinical study analysis. J Cataract Refract Surg 33: 1217-1226.
2. Lewis RA, von Wolff K, Tetz M, et al (2009) Canaloplasty: circumferential viscodilation and tensioning of Schlemm canal using a flexible microcatheter for the treatment of open-angle glaucoma in adults. Two-year interim clinical study analysis. J Cataract Refract Surg 35:814-824.

3. Grieshaber MC, Pienaar A, Olivier J, Stegmann R (2010) Canaloplasty for primary open-angle glaucoma: long-term outcome. Br J Ophthalmol 94: 14781482.

4. Koerber NJ (2012) Canaloplasty in one eye compared with viscocanalostomy in the contralateral eye in patients with bilateral open-angle glaucoma. J Glaucoma 21: 129-134.

5. Lewis RA, von Wolff K, Tetz M, Koerber N, Kearney JR, et al. (2011) Canaloplasty: Three-year results of circumferential viscodilation and tensioning of Schlemm canal using a microcatheter to treat open-angle glaucoma. J Cataract Refract Surg 37: 682-690.

6. Bull H, von Wolff K, Körber N, Tetz M (2011) Three-year canaloplasty outcomes for the treatment of open-angle glaucoma: European study results. Graefes Arch Clin Exp Ophthalmol 249: 1537-1545.

7. Brusini P, Caramello G, Benedetti S (2013) Canaloplasty in open angle glaucoma. Mid-term results from a multicenter study. J Glaucoma, in press.

8. Grieshaber MC, Fraenkl S, Schoetzau A, Flammer J, Orgül S (2011) Circumferential viscocanalostomy and suture canal distension (canaloplasty) for whites with open-angle glaucoma. J Glaucoma 20: 298-302.

9. Grieshaber MC, Pienaar A, Olivier J, Stegmann R (2010) Comparing two tensioning suture sizes for 360 degrees viscocanalostomy (canaloplasty): a randomised controlled trial. Eye (Lond) 24: 1220-1226.

10. Brusini P, Tosoni C (2014) Canaloplasty after failed trabeculectomy: a possible option. J Glaucoma 23: 33-34.

11. Ayyala RS, Chaudhry AL, Okogbaa CB, Zurakowski D (2011) Comparison of surgical outcomes between canaloplasty and trabeculectomy at 12 months' follow-up. Ophthalmology 118: 2427-2433.

12. Brüggemann A, Müller M (2012) [Trabeculectomy versus canaloplasty--utility and cost-effectiveness analysis]. Klin Monbl Augenheilkd 229: 1118-1123. 